\title{
Lasers in Prosthetics
}

Deepak Thomas ${ }^{1}$

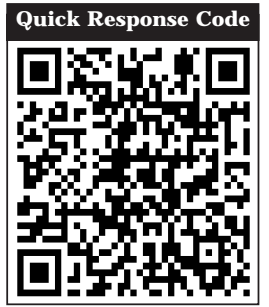

doi : $10.5866 / 2014.631600$

Prosthodontist \& Implantologist

Department of Dentistry

Indira Gandhi Medical College \& Research Institute Vazhudavur Road, Kadirkamam

Puducherry 605009, India.

\section{Article Info:}

Received: April 8, 2014

Review Completed: May 9, 2014

Accepted: J une 7, 2014

Available Online: October, 2014 (www.nacd.in)

(C) NAD, 2014 - All rights reserved

Email for correspondence:

deepak32@gmail.com

\section{ABSTRACT:}

In this changing era all the disadvantages of the conventional methods are being overcome by newer technology like lasers. Lasers are introduced in every field of dentistry including prosthodontics. Lasers are widely used in every aspects of Prosthodontics. The aim of this article is to elaborate the applications and uses of the lasers in Prosthodontics.

Key words: Lasers, prosthetics, removable, fixed, implants.

\section{INTRODUCTION}

Laser dentistry, formerly embraced only by the speciality of oral and maxillofacial surgery, now, is positively affecting every field of dentistry. From pediatric and operative dentistry to periodontics, prosthetics to cosmetics and implantology, lasers have made a tremendous impact on the delivery of dental care in the $21^{\text {st }}$ century and will continue to do so as the technology continues to improve and evolve.

The word laser is an acronym for "Light Amplification by stimulated Emission of Radiation" which explains most of the critical physical interactions that occur within a laser generating cavity. This property of radiant energy reaction with biol ogical tissues has been taken as the basis of laser treatment in dentistry. ${ }^{1}$

In early 1960s, the first working laser was invented by Theodore Maiman who inserted a ruby rod into a photo-graphic flashlamp. In 1964, Ralph Stern and Reidar Sognnaes used the ruby laser to vaporise enamel and dentine. In 1969 Leon Goldman used the laser clinically on enamel and dentine. Initially, application of lasers for dental use was tested for hard tissue, but surface cracking and thermal damage to the enamel and dentine were reported. It is not until 1989 when the first dental laser was developed, a 3W neodymium-doped yttrium aluminium garnet ( $\mathrm{Nd:YAG)} \mathrm{by} \mathrm{Drs} \mathrm{Terry}$ and Bill Meyers, for soft tissue use, since that time 
a variety of laser wavelengths have been introduced and marketed.

\section{Lasers in Prosthetics}

Lasers are now being used in a variety of procedures in prosthetic dentistry.

\section{Complete Denture Prosthetics}

A) Prototyping and CAD/CAM technology: The term rapid prototyping (RP) refers to a class of technologies that can automatically construct physical models from Computer-Aided Design (CAD) data. These "three dimensional printers" allow designers to quickly create tangible prototypes of their designs, rather than just twodimensional pictures. Such models have numerous data.In addition to prototypes, RP techniques can also be used to make tooling (referred to as rapid tooling) and even production-quality parts (rapid manufacturing). A software package slices the CAD model in to a number of thin (eg. $0.1 \mathrm{~mm}$ ) layers, which are then built up one atop another. Rapid prototyping is an additive process, combining layers of paper, wax, or plastic to create a solid object. In contrast, most machining processes (milling, drilling, grinding, etc.) are "subtractive" processes that remove material from a solid block. RP's additive nature allows it to create objects with complicated internal features that cannot be manufactured by other means. ${ }^{2,3}$

\section{i. Laser Rapid forming of a Complete} Titanium Denture Base Plate: This technique uses the combination of the CAD/CAM and LRF (Laser Rapid Forming) methods for forming the titanium plate of a complete denture. Laser scanner, reverse engineering software, and standard triangulation language (STL) formatted denture base plate and sliced into a sequence of numerical controlled codes.The denture plate will be built layer-by-layer, on the LRF system. After the traditional finishing techniques, this denture plate will be acceptable for use in patients. ${ }^{4}$

B) Analysis of Occlusion by CAD/CAM: After fabrication of new dentures the occlusion can be examined and studied with the help of laser scanner technique and three-dimensional reconstruction. The relationship between the parameters of balanced occlusion can also be analyzed. ${ }^{5}$

C) Analysis of Accuracy of I mpression by Laser Scanner: Several studies have made comparisons in the dimensional accuracy of different elastomeric impression materials. Most have used two dimensional measuring devices, which neglect to account for the dimensional changes that exist along a three-dimensional surface. The scanning laser three-dimensional (3D) digitizer can delineate $x, y$, and $z$ coordinates from a specimen without actually contacting the surface. The digitizer automatically tracks coordinates with precision and stores data as the number of points on a surface with a resolution of $130 \mathrm{~mm}$ at $100 \mathrm{~mm}$. These exacting features suggest that the laser digitizer might accurately and reliably measure the dimensions of dental impression materials while avoi ding subjective errors. The image is built up and landmarks identified which allow superimposition of the images and so enable the differences between two similar images to be calculated. The 3D laser captures complex 3D texture-mapped models and they are exported into a 3D (Scan Surf) software application where it is built and triangulated into a 3D meshwork image of the object. The scanning process is accomplished within a minute whereas the software analysis takes much longer. The software superimposes the two objects by either registering landmarks or by registering as iterative closest point (ICP). This finds an optimal fit between the two surfaces and in effect acts as a reference area. Once superimposed, the difference of the two surfaces is cal culated as the shortest distance of each point on one object surface from a second object surface, within a range of $0.5 \mathrm{~mm}$. Threedimensional digitizers will eventually become less expensive, require less maintenance, track faster, and be available with more standardized software. ${ }^{6}$

\section{Removable Dentures Prosthetics}

A. Treatment of unsuitable alveolar ridges: Alveolar resorption usually is uniform in vertical and lateral dimensions. To smooth the residual ridge, to expose the bone soft tissue lasers 
surgery may be performed with any number of soft tissue wavelengths (CO2, diode, Nd:YAG,) Hard tissue surgery may be performed with the erbium family of wavelengths.

\section{B. Treatment of undercut alveolar ridges:} There are many causes of undercut alveolar ridges. Naturally occurring undercuts such as those found in the lower anterior alveolus or where a prominent pre-maxilla is present may bethe cause of soft tissue trauma, ulceration, and pain when prosthesis is placed on such a ridge. Soft tissue surgery may be performed with any of the soft tissuelasers. Osseous surgery may be performed with the erbium family of lasers.

C. Treatment of enlarged tuberosity: The most common reason for enlarged tuberosities usually is soft tissue hyperplasia and alveolar hyperplasia accompanying the over-eruption of unopposed maxillary molar teeth. Surplus soft tissue should be excised, allowing room for the denture bases. The soft tissue reduction may be performed with any of the soft tissue lasers. Erbium laser is the laser of choice for the osseus reduction.

D. Surgical treatment of tori and exostoses: Prosthetic problems may arise if maxillary tori or exostoses are large or irregular in shape. Soft tissue lasers may be use to expose the exostoses and erbium lasers may be use for the osseous reduction. A smooth, rounded, midlinetorus normally does not create a prosthetic problem because the palatal acrylic may be relieved or cut away to avoid the torus.

E. Soft tissue lesions: Persistent trauma from a sharp denture flange or over compression of the posterior dam area may produce a fibrous tissue response. Hyperplastic fibrous tissue may beformed at the junction of the hard and soft palate as a reaction to constant trauma and irritation from the posterior dam area of the denture. The lesion may be excised with any of the soft tissue lasers and the tissue allowed to re epithelialize. ${ }^{7}$

F. Laser welding: One of the modern methods of removable partial dentures defect repairs uses the pulsed laser with relative low average out power. This is known as a precise and rapid joining method, but its success depends on the control of many parameters. Eg: For Co-Cr alloy framework. ${ }^{8}$

\section{Fixed Denture Prosthetics}

A. Crown lengthening: The advantage of lasers in crown lengthening is that it cuts only at the tip and they can be held parallel to long axis of the tooth to remove bone immediately adjacent to cementum without damaging it. ${ }^{9} \mathrm{H}$ ence lasers offer extreme precision and operator control for fine tracing of incision lines and sculpting of the desired gingival margin outline.

B. Soft tissue management around abutments: Argon laser energy has peak absorption in hemoglobin, thus lending itself to providing excellent hemostasis and efficient coagulation and vaporization of oral tissues. These characterisitics are beneficial for retraction and hemostasis of the gingival tissue in preparation for an impression during a crown and bridge procedure. Gingivoplasty may also be done using argon laser. ${ }^{10}$

C. Osseous crown lengthening: Like teeth mineralized matrix of bone consists mainly of hydroxyapatite. The water content and hydroxyapatite are responsible for the high absorption of the Er: YAG laser light in the bone. Er: YAG laser has very promising potential for bone ablation. ${ }^{10}$

D. Troughing: Lasers can be used to create a trough around a tooth beforeimpression taking. This can entirely replace the need for retraction cord, electrocautery, and the use of hemostatic agents. The results are predictable, efficient, minimize impingement of epithelial attachment, cause less bleeding during the subsequent impression, reduce postoperative problems, and reduce chair time. It alters the biological width of gingiva. Nd:YAG laser is used. ${ }^{10}$

E. Formation of ovate pontic sites: For favorable pontic design recontouring of soft and bony tissue may be needed. Soft tissue surgery may be performed with any of the soft tissue lasers and osseus surgery may be performed with erbium family of lasers. ${ }^{11}$ 
F. Veneer removal: With laser technology, the restoration can now be removed without cutting it off. The laser energy passes through porcelain glass unaffected and is absorbed by the water molecules present in the adhesive. It appears that this debonding occurs at the silane-resin interface because the underlying tooth structure appears to be unaffected. The lasers used are Er:YAG or Er and $\mathrm{Cr}$ : YSGG.

G. Altered passive eruption management: Lasers can be used very efficaciously to manage passive eruption problems . when the patients have clinical crowns that appear too short or when they have an uneven gingival line producing an uneven smile, excessive tissue can be easily and quickly removed without the need for blade incisions, flap reflection, or suturing.

H. Modification of soft tissue around Iaminates: The removal and recontouring of gingival tissues around laminates can be easily accomplished with the argon laser. ${ }^{12}$

I. Crown fractures at the gingival margins: Er:YAG or Er, Cr:YSGG lasers can be carried out to allow correct exposure of the fracture margin. ${ }^{13}$

J. Crown Preparation: Crown preparation with lasers a debated topic still. There are no conclusive studies yet showed the use of lasers for crown preparation purposes. But still some commercial companies say that they can be used. $\mathrm{Er}, \mathrm{Cr}$ : YSGG laser is used most commonly now. It uses hydrokinetic technology (laser-energized water to cut or ablate soft and hard tissue).Because of this mechanism local anesthesia is not required in many cases, making this more comfortable procedure for the patient, and of course, saving time and anesthetic use by the patient. For vital crown preparation no need of local anesthesia, as laser causes temporary paresthasia of nerve endings. Procedure is accurate and faster than the conventional method. ${ }^{14}$

\section{Implantology}

A. Implant recovery: One advantage of use of lasers in implantology is that impressions can be taken immediately after second stage surgery because there is little blood contamination in the field due to the haemostatic effects of the lasers. There also is minimal tissue shrinkage after laser surgery, which assures that the tissue margins will remain at the same level after healing as they are immediately after surgery. ${ }^{15}$

B. Implant site preparation: Lasers can be used for the placement of mini implants especially in patients with potential bleeding problems, to provide essentially bloodless surgery in the bone. ${ }^{16}$

C. Removal of diseased tissue around the implant: Lasers can be used to repair ailing implants by decontaminating their surfaces with laser energy. Diode, CO2 \& Er:YAG lasers can be used for this purpose. Lasers can also be used to remove granulation tissue in case there is inflammation around an already osseointegrated implant. ${ }^{17,} 18$

D. Sterilization of socket: In immediate implant dentistry after extraction of tooth, without any infection, socket can be sterilized immediately without any pain.

E. Peri Implantitis: Since the laser does not transmit damaging heat, it can be utilized to vaporize any granulation tissue as well as clean the implant surface in peri-implantitis cases. This procedure eliminated the acute state of periimplantitis, resulting in positive GTR, and allowing the patient extended use of the implant. ${ }^{19,} 20$

F. Sinus lift procedure: Lasers can also be used in the sinus lift procedure. The procedure can be done by making the lateral osteotomy with a decreased incidence of sinus membrane perforation. Theyttrium-scandium-gallium-garnet (YSGG) laser is the optimal choice for not cutting the sinus membrane.The YSGG laser can al so be used to make the osteotomy for a ramal or symphyseal block graft. Bone grafts done with lasers have been demonstrated to decrease the amount of bone necrosis from the donor site and the osteotomy cuts are narrower, resulting in less postoperative pain and edema. ${ }^{21}$ 


\section{Maxillofacial Prosthetics}

New advances in rapid prototyping technologies have demonstrated significant advantages compared to more conventional techniques for fabricating facial prosthesis. The use of selective laser sintering technology is an alternative approach for fabricating a wax pattern of maxillofacial prosthesis. This new approach can generate the wax pattern directly and reduce labor-intensive laboratory procedures.22

\section{Lab Procedures}

Lasers have been used for deposition of hydroxyapatite (HA) thin films on titanium implants. Pulsed laser deposition (PLD) has proven to be a promising method to produce pure, crystalline and adherent HA coatings which show no dissolution in a simulated body fluid. Use of lasers for surface treatment of titanium castings for ceramic bonding have shown improved bond strength when compared to acid etching techniques which are commonly used. Lasers can also be used for welding. ${ }^{23}$

\section{Conclusion}

The use of laser in daily clinical work is slowly becoming a reality, hel ping to correct the view that laser procedures are too sophisticated and lead to higher costs without the corresponding benefits. It is to be clearly understood that the usage of laser in prosthetics has so many advantages like precision, ease of operation, patient comfort, time reduction etc. So it is the need of the hour to incorporate laser to fullest extent in dentistry which in turn will lead not only to the modernization of treatment but wellness of mankind.

\section{References}

1. Donald J Coluzzi: an overview of laser wavelengths used in dentistry: DCNA 2000; 44(4):753-766.

2. Richard J. Thomas. Method for automatically creating a denture using laser altimetry to create a digital 3-D oral cavity model and using a digital internet connection to a rapid steriolethographic modeling machine. United States patent 2006; US 7,153,135 B1.

3. Sun YC, Lu PJ , Wang Y et al. Research and development of computer aided and rapid prototyping technology for complete dentures. Chinese J ournal of Stomatology 2007; 42(6):324-329.

4. Wu J, Gao B, Tan H et al. A feasibility study on laser rapid forming of a complete titanium denture base plate. Lasers Med Sci 2008.
5. E Y Lu, F Q Zhang, X J Chen et al. A study of complete denture e occlusion using by three-dimensional technique. IADR exhibition 2006.

6. Sinal Shah, Geeta Sundaram, David Bartlett et al. The accuracy of a 3D laser scanner using superimposition software to assess the accuracy of impression techniques. J Dent 2004; 32:653-658.

7. Goldman L,Gray J A, Goldman J , Goldman B, Meyer R, Effects of laser impacts on teeth. J Am Dent Assoc 1965; 70:601-606.

8. Liliana sandu, Birdeanu V. Laser welding procedures applied to removable partial denes framework repairs. Eur Cells Mat 2006; 11(2):29.

9. K. R. Nagaraj. Use of lasers in prosthodontics: A review. Int J Clin Dent 2012; 5(1):91-112.

10. Leo J .Miserendino and Robert M Pick. Lasers in dentistry 1995; 133-168.

11. J Ramya J yothy,Lasers in prosthetic dentistry. Ind J Applied Res 2013; 3(4):369-371.

12. Vikas Punia, Vivek Lath, Meenakshi Khandelwal, Sandhya Kapoor Punia, Rohit Lakhyani. The current status of laser application in Prosthodontics. NJ IRM 2012; 3(3): 170-175.

13. Parker S. Lasers and soft tissue: 'fixed' soft tissue surgery. Br Dent J 2007; 202:247-253.

14. Dr.Rosh nash. Crown and veneer preparation using the $E r$, Cr:YSGG Waterlase ${ }^{\mathrm{TM}}$ hard and soft tissue laser. Cont Esth Rest Prac 2002; 80-85.

15. Manni JG. Dental applications of advanced lasers, Barlington(VT): J GM associates; 1996.

16. Strauss R. Lasers in oral and maxillofacial surgery. Dent Clin N Am 2000; 44(4):861-888.

17. Coluzzi DJ .Dent Clin North America 2004; 48:999-1015.

18. Kato T, Kusakari $\mathrm{H}$ et al. Bactericidal efficacy of $\mathrm{CO} 2$ laser against bacteria contaminated titanium implant and subsequent cellular adhesion to irradiated area. Lasers Surg Med 1998; 23(5):299-309.

19. Stefan stubinger, Frank homann. Effect of Er: YAG, CO2 and diode laser irradiation on surface properties of zirconia endosseous dental implants. Laser Surg Med 2008; 40(3):223-228.

20. Miller, Robert J. Treatment of the contaminated implant surface using the $\mathrm{Er}, \mathrm{Cr}$; YSGG Iaser. Implant dentistry 2004; 13(2):165-170.

21. Convissar RA,Gharemani EH. Laser treatment as an adjuvant to removable prosthetic care. Gen Dent 1995; 43:44.

22. Guofeng Wu, Bing Zhou. Selectivelaser sintering technol ogy for customized fabrication of facial prostheses. J Prosthet Dent 2008; 100:56-60.

23. C. Bertrand, Y. Le Petitcorps, L. Albingre, V. Pupis. The laser welding technique applied to the non-precious dental alloys procedure and results. Bri Dent J 2001; 190(5):225257. 